\title{
Sunspot Activity, Influenza and Ebola Outbreak Connection
}

Jiangwen $\mathbf{Q u}^{1}$, Zhigang Gao1, Ying Zhang ${ }^{1}$, Milton Wainwright ${ }^{2}$, Chandra Wickramasinghe ${ }^{3,4}$ and Tareq Omairi $^{2}$

${ }^{1}$ Department of Infectious Disease Control, Tianjin Center for Disease Control and Prevention, China

${ }^{2}$ Department of Molecular Biology and Biotechnology, University of Sheffield, Sheffield S10 2TN, UK

${ }^{3}$ Buckingham Centre for Astrobiology [BCAB], Buckingham University, UK

${ }^{4}$ Churchill College, Cambridge CB3 OHA, UK

"Corresponding author: Wickramasinghe NC, Buckingham Centre for Astrobiology [BCAB], Buckingham University, Churchill College, Cambridge CB3 OHA, UK, Tel: +44 (0)2920752146, +44 (0)7778389243; E-mail: ncwick@gmail.com

Received date: August 11, 2016; Accepted date: August 24, 2016; Published date: August 31, 2016

Copyright: ( $2016 \mathrm{Qu} \mathrm{J}$, et al. This is an open-access article distributed under the terms of the Creative Commons Attribution License, which permits unrestricted use, distribution and reproduction in any medium, provided the original author and source are credited.

\begin{abstract}
An ever-present threat of a major new influenza pandemic of devastating proportions haunts us, and despite diligent monitoring of circulating viruses has been impossible to predict. On a more local scale outbreaks of Ebola Hemorrhagic Fever (EHF) in West Africa have posed a serious public health threat because of its high fatality risk. Research on the environmental factors underlying both EHF and influenza epidemiology may provide useful insights into the occurrence of future pandemic outbreaks. This study suggests that sunspot activity in extremum or \pm one year was associated with influenza pandemics and severe EHF outbreaks in Africa from 1976 to 2014 . Potential mechanisms by which sunspot activity may influence viral outbreaks are discussed. Current and future surveillance efforts should be supported to construct a comprehensive early warning system involving virus monitoring and epidemioilogy on the ground, sunspot activity as well as stratospheric sampling for forecasting future pandemics.
\end{abstract}

Keywords: Influenza; Ebola; Microbial Ecology; Sunspots; Cosmic environment

\section{Introduction}

Of the many types of global disasters that face humanity at the present time the threat of a major viral pandemic would appear to be among the most serious. Despite the huge strides of progress that have been made in regard to studies of influenza $\mathrm{A}$ and the immunization strategies that are in place, we are still unable to predict the emergence of new pandemic strains, forecast the onset of a pandemic or plan reliable steps to ameliorate its worst outcome.

The famous 1918-1919 H1N1 pandemic caused an estimated 30 million deaths worldwide. The human and economic cost of another pandemic on a similar scale will be difficult to assess. Although a close monitoring of newly emerging recombinant strains has been in progress under the auspices of $\mathrm{WHO}$ as a coordinating body the conditions required for pandemic spread have not been possible to predict. Likewise, it has not been possible to predict or understand properly the onset of pandemics of other viral diseases, notably Ebola and Zika.

Recent studies involving DNA sequencing of humans and primates have shown the preponderance of sequences derived from retroviruses that make up a significant $8 \%$ of the human and primate genomes. It is likely that these sequences were inserted as retroviruses into our ancestral DNA through a long sequence of pandemics of disease caused by RNA viruses. It is also probable that such viral DNA played a crucial role in hominid evolution over millions of years [1]. In particular retroviral sequences from the Ebola virus family have recently been discovered in several mammalian species including humans, indicating that these viruses have been circulating for tens of millions of years [2]. Whether these ancient RNA viruses were indigenous to our planet or derived from an external cosmic source cannot of course be resolved.

\section{Panspermia context}

Fred Hoyle and one of the present authors developed the theory that new bacteria, viruses and virions are continually resupplied to the Earth from the external cosmos, in particular from comets [1]. Whilst this Hoyle-Wickramasinghe theory might be seen as unorthodox, its plausibility has been enhanced in recent years with new data from geology and astronomy. The discovery of graphitized remnants of microbes in 4.1 billion-year old rocks by Bell et al. [3] provides strong evidence for the first microbial life arriving at the Earth via impacting comets. Recent studies of comets, in particular comet 67P/C-G have shown evidence for the products of microbial metabolism, including high levels of elemental P consistent with RNA/DNA [4].

Comets, of which there are some 100 billion in our solar system, are typically $10 \mathrm{~km}$ sized frozen bodies comprised mostly of water-ice and organic materials, the latter most probably associated with life. It is taken for granted that these objects carry the complex building blocks of life. More controversially they are also considered to harbor microbial life in radioactively heated subsurface domains. Eruptions of such domains were observed in the case of come 67P/C-G to contain tantalizing signs of biological material that would inevitably have had the capability of interacting with the terrestrial biosphere.

Zhang et al. [5] have recently shown that a vast abiotic reservoir of Influenza A exists in the form of viral genes frozen in ice in high latitude lakes on the Earth. Ice and water from Siberian lakes showed positive for influenza A $\mathrm{H} 1$ genes. Although Zhang et al. attribute a fecal source of virus from migratory waterfowl; their results are clearly compatible with the possible existence of a source of influenza genes that can persist outside an animal reservoir, even perhaps in comets. It is possible to think of frozen commentary lakes containing a gigantic 
reservoir of RNA viruses some of which can periodically gain ingress to the Earth.

The extension of the terrestrial biosphere beyond the troposphere, even to heights above $20 \mathrm{~km}$, seems now to be beyond dispute. Sampling of the stratosphere carried out since 2001 have confirmed the existence of micro biota up to heights of $41 \mathrm{~km}$, and these include 10 micron-sized clumps of putative bacterial and viral material that could not have been lofted from the Earth's surface [5,6]. A total daily input of 0.1 tonnes of viral and microbial material has been inferred as falling to the Earth from outer space, and most of this material is believed to be of cometary origin [5]. If this is accepted a thorough analysis of all data relating to such input is clearly of crucial importance in assessing its effect on terrestrial life.

Critics might argue that it is impossible for microorganisms, such as bacteria and viruses, to make the long journey (both in time and space) from comets and other cosmic sources to Earth. However, compelling evidence has recently been obtained by Wainwright and Omari [7] which shows that biological entities, which appear to have no Earth analogues, are continually incoming to Earth from space. Such evidence obviously goes against the a priori assumption that panspermia is impossible and that that life is restricted to Earth.

\section{Diseases from space}

It was first pointed out by Hoyle and Wickramasinghe [8] that many aspects of historical pandemics appear to be fully consistent with microbial agents delivered from sources external to the Earth. In such a scheme the evolution of terrestrial life, including humans, occurred in response to a continual accommodation of bacterial and viral genes from comets. Whilst a virus like influenza A undergoes continual evolution and host adaptation through internal processes- mutation and recombination-there is growing evidence to suggest a contribution from a more important effect that is still not understood. We shall argue that the emergence of new pandemic strains appears to involve a cosmic causal connection.

Any possible connection between pandemics of disease and the external universe has for a long time been viewed with suspicion in Western scientific culture. But this is not so in the cultural traditions of either China or India. In view of the enormous global economic repercussions of any future pandemic it is prudent to set aside cultural constraints and follow the scientific facts alone. In China we keep an open mind on these matters and propose to take whatever steps may be needed to enable prediction/forecasting of future pandemics and to initiate action to ameliorate their worst effects.

\section{Association between influenza and sunspot activity}

We now summarise influenza data that have already been discussed at length by $\mathrm{Qu}$ [9] elsewhere, and we refer the reader to this work for further details and citations. In the past half century, sporadic cases of severe influenza and deaths in humans have been caused by a number of animal influenza A viruses, including the Hsw1N1virus, first detected in 1976, the H5N1 virus, first detected in 1997, and the H7N9 virus, first reported in 2013. The World Health Organization (WHO) has flagged such sporadic cases as possible harbingers of an impending pandemic but thus far they have not turned out to be so.

In early 1976, the novel A/New Jersey/76 (Hsw1N1) influenza virus caused severe respiratory illness in 13 soldiers with 1 death at Fort Dix, New Jersey. Since the subtype A/New Jersey was similar to the 1918-
1919 pandemic virus, rapid outbreak assessment and enhanced surveillance were initiated, but fortunately no pandemic ensued. During an outbreak of a highly pathogenic avian influenza (HPAI) H5N1 in poultry markets in Hong Kong in 1997, 18 people were infected, six of whom died. This was the first recorded direct transmission of an avian influenza virus to humans causing fatality. Again no pandemic happened. On March 30, 2013, a novel avian influenza A H7N9 virus causing human infections was identified in China. As of April 18, 2013, the virus had spread to six provinces and municipal cities-i.e., Shanghai, Anhui, Jiangsu, Zhejiang, Beijing, and Henan. As far as we know this outbreak represents the first time that the H7N9 subtype has infected humans and caused fatal cases (as of April 18, 2013, 87 people have been infected and 17 have died).

Point mutations in RNA produce relatively minor changes/genetic drifts of the influenza virus on a more or less continual basis whilst the virus circulates. The emergence of pandemic strains, however, apparently requires radical genetic transformations or shifts involving a major re-assortment of viral genes, including recombination of preexisting influenza sub-types, and the possibility already discussed that new RNA segments may be inserted that have an origin external to Earth. In the latter case changes in the Earth's electromagnetic environment, which is in turn controlled by the solar magnetic field and the solar wind, may have an effect in the delivery of virions of cometary origin. The sun's magnetic field reverses through the solar cycle, and it may be reasonable to expect more efficient transport of virions from the stratosphere to the ground at times of solar maxima and minima [10]. Particularly large individual sunspots may have an important role to play in this context. Although the possibility of externally derived virions may also be considered controversial, it is consistent with aspects of influenza pandemic epidemiology that cannot otherwise be explained [11].

Weinstein [11], who studied the data on the 1918-1919 pandemic wrote thus: "It was detected in Boston and Bombay on the same day, but took three weeks before it reached New York City, despite the fact that there was considerable travel between the two cities..." Person-toperson transmission at such a speed would not have been possible in 1918 before the advent of air travel.

\section{Sunspot connection}

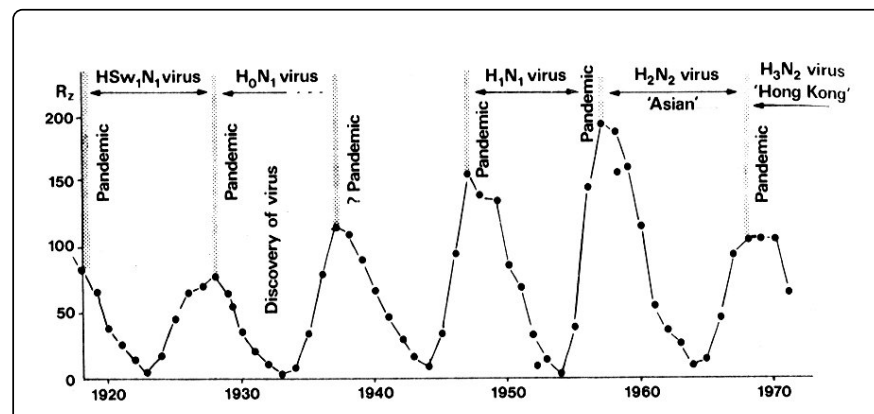

Figure 1: Hope-simpson's correlation of sunspot peaks and pandemics [12].

A connection between the appearance of pandemic outbreaks and sunspots was first pointed out by Hope-Simpson [12]. Almost every influenza pandemic of the $20^{\text {th }}$ century has occurred at times that coincided with peaks in the sun's 11 year cycle of sunspot activity, and a similar relationship may even extend through into the $18^{\text {th }}$ century. 
The picture upto 1972 is shown in Figure 1, noticing also that the pandemic of the H1N1 strain in 1977 came close to the next peak in this sequence. The inference would appear to be that pandemics of influenza are unlikely to start at times significantly removed from a peak of the sunspot cycle.

$\mathrm{Qu}[9]$ has studied the sunspot-influenza correlation more carefully and concluded that not only peaks in the sunspot cycle, but minima are also sometimes relevant. It is concluded that the onset of pandemics and possible pandemics coincide with sunspot extrema [maxima and minima] to within \pm 1 year. This is shown in Figure 2 .

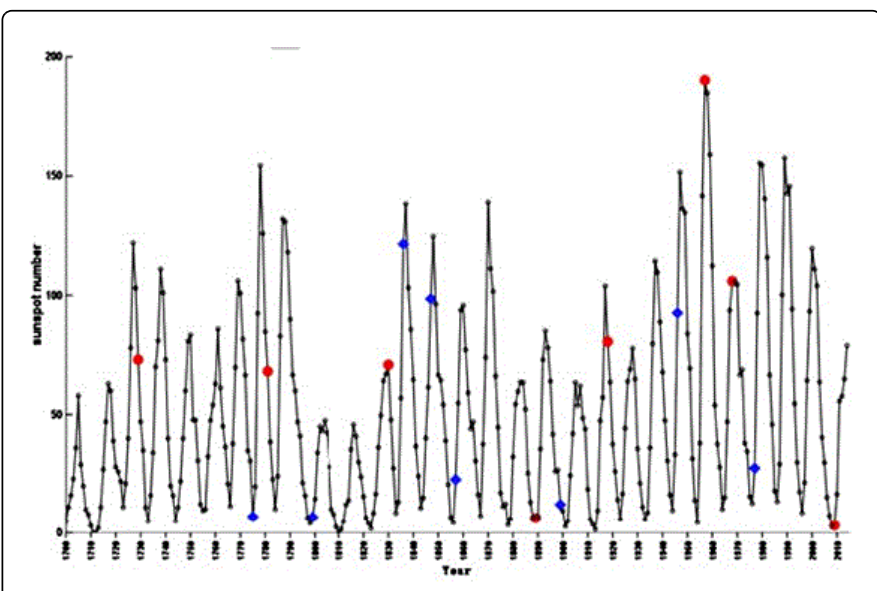

Figure 2: The association between sunspot cycles and influenza pandemics, 1700-2014 A.D. Red circles represent the starting years of definite influenza pandemics; blue triangles represent the starting years of possible influenza pandemics [9].

The 1976 Hsw1N1 influenza outbreak occurred in sunspot minimum year, The 1997 H5N1 influenza outbreak occurred in sunspot minimum plus 1 year, whereas the 2013 H7N9 influenza outbreak occurred in sunspot maximum minus 1 year. These facts seemed to suggest extremes of sunspot activity to within \pm 1 year were risk factors of influenza pandemics.

\section{Association between severe Ebola outbreaks and sunspot activity}

The rapid spread of Ebola hemorrhagic fever (EHF) in West Africa has become a major threat and challenge to public health on both local and global scales. The World Health Organization (WHO) declared the outbreak to be a public health emergency of international concern on 8 August 2014. Although medical professionals worldwide are attempting to study how Ebola outbreaks occur, the key reasons driving the emergence of EHF remain poorly understood. With the eventual control of 2014 Ebola outbreak, this infectious disease can be expected to remain a considerable challenge for the foreseeable future. It is prudent to equip ourselves as best we can to cope with any future resurgence of the disease, and in particular to discover what factors might precipitate its emergence.

Unlike influenza which is globally prevalent with a wide distribution of host species-humans, birds, pigs-Ebola is geographically localized and confined to a locally defined set of hosts. Fruitbats of the Pteropodidae family are generally thought to be the natural host/ carrier of Ebola, and susceptible animals include primates (monkeys, gorilla, apes), antelope and porcupines. Geographical and species localization are, however, no barrier to changes to the viral genome that can suddenly be wrought due to hybridization/recombination events with globally prevalent or space-incident viral genes. Recombination could occur with other related and compatible viruses or virions that could be a global phenomenon driven, as in the case of influenza [9], by the sunspot cycle.

The Ebola data for our study were obtained from Centres for Disease Control and Prevention, available at http://www.cdc.gov/vhf/ ebola/outbreaks/history/Chronology.html Sunspot number data were provided by WDC-SILSO, Royal Observatory of Belgium, Brussels (http://www.sidc.be/silso/versionarchive).

A total of 29 reported EHF outbreaks in Africa were identified from references and records listed on the United States Centers for Disease Control and Prevention website. Severe Ebola outbreaks in which the total number of cases was $\geq 100$ between 1976 and 2013 occurred in $1976,1995,2000-2001,2001-2002,2002-2003$, and 2007-2008, with the number of cases being $602,315,425,122,163$ and 413, respectively. The outbreaks in question were caused by different viral subtypes.

The outbreak in 2014 was the largest and deadliest since the disease was first recognized in 1976 in northern Zaire. As of 28 December 2014, the WHO reported 20206 cases (including laboratory-confirmed, probable, and suspected cases) and 7905 deaths in nine countries. A potential explanation was explored by comparing solar activity with the timing of the emergence of severe EHF outbreaks. The starting dates of severe Ebola outbreaks are shown by the red triangles in Figure 3. The severe Ebola outbreaks in 2000-2001, 2001-2002, and 2014 occurred at sunspot maximum or \pm one year, whereas the outbreaks in 1976, 1995, and 2007-2008 occurred in sunspot minimum or \pm one year. It is interesting to note that 3 of the 5 peak dates of Ebola correspond closely to peaks in pandemic influenza. Detailed statistical analysis of this data is discussed in Appendix 1.

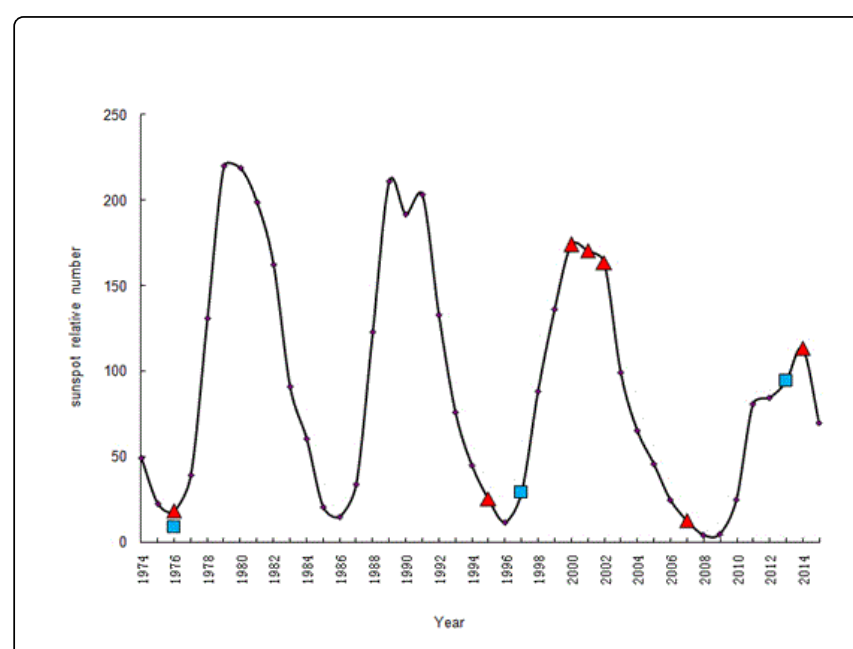

Figure 3: The association between sunspot activity and potential influenza pandemics and severe Ebola outbreaks, 1974-2015. Blue squares represent the starting years of potential influenza pandemics. Red triangles represent the starting years of potential influenza pandemic and severe Ebola outbreak. 


\section{Possible mechanisms associated with sunspots affecting Influenza and Ebola}

The cosmic ray intensity at the Earth's surface falls on the average during maximum sunspot activity and rises during minimum sunspot activity (Figure 4). The result of linear correlation analysis indicated that the coefficient of correlation between relative sunspot number and cosmic ray data was $-0.90(\mathrm{P}=0.000)[9]$.

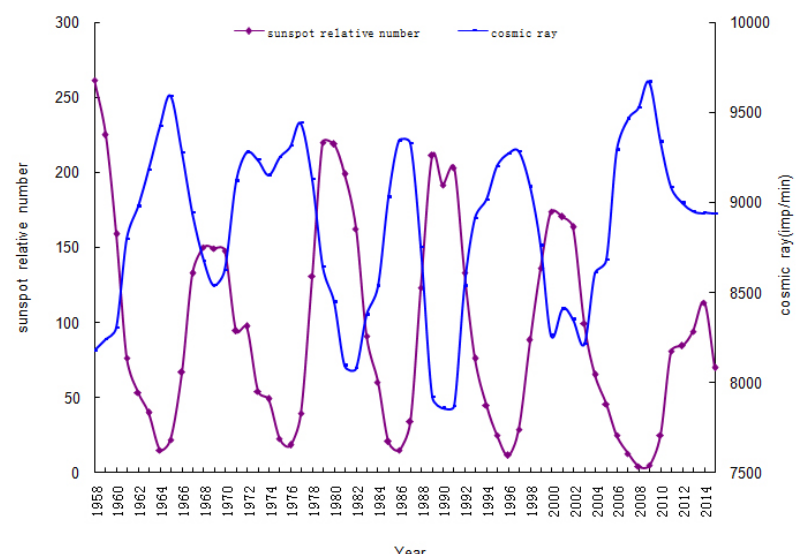

Figure 4: Comparison between sunspot numbers and cosmic ray flux at Earth's surface from 1967-2016 [9].

Potential influenza pandemics and severe Ebola outbreaks being confined to particular years, we infer that some special or unusual factors must have emerged and played decisive roles. Our study has shown that sunspot activity in extremum (maximum or minimum) \pm one year was likely to be one of the factors in the development of potential influenza pandemics and severe Ebola outbreaks. Sunspot numbers are strongly correlated with solar activity, such as X-ray flares. Increased numbers of solar flares and coronal mass ejections produce increased fluxes of high-energy solar particles during the maxima [13-15]. Furthermore, cosmic rays reach a maximum intensity when the sun is least active and fall at minimum intensity during the solar maximum period [14]. Our results also indicated that the coefficient of correlation between the relative sunspot number and cosmic ray data was -0.90 .

Point mutations, gene recombination, and gene re-assortment are thought to be the three basic mechanisms of enhanced infectivity and virulence of viral agents [16]. Solar particles and cosmic rays are physical entities capable of causing point mutations, and can be thought to lead to the emergence of pandemic influenza potential and severe Ebola outbreaks. Re-assortment is fundamental to the evolution of influenza viruses and plays a key role in the generation of epidemiologically significant strains. For example, H5N1 viruses that infected humans in Hong Kong in 1997 were probably re-assortments that derived their $\mathrm{H} 5$ gene from a goose virus related to AyGooseyGuangdongyly96 (H5N1) and their internal gene complex from a QayHKyG1y97-like virus. The origin of the N1 neuraminidase is related most closely to the NA gene of the A/Parrot/Ulster/73 (H7N1) virus $[17,18]$. The novel avian influenza A H7N9 virus originated from multiple re-assortment events. The HA gene might have originated from avian influenza viruses of duck origin, and the
NA gene might have transferred from migratory birds infected with avian influenza viruses along the east Asian flyway. The six internal genes of this virus probably originated from two different groups of H9N2 avian influenza viruses, which were isolated from chickens $[19,20]$. The re-assortment is an important evolutionary mechanism which can result in antigenic shifts that modify host range, pathology, and transmission of the influenza A viruses.

Recombination and re-assortment often occur at highly variable frequencies in viruses with RNA as their genetic material. Multiplicity reactivation and cross reactivation have been proven to be important mechanisms of genetic recombination and re-assortment. Reactivation has been observed in influenza viruses irradiated by all types of radiations, such as ultraviolet light and gamma ray, in laboratories during the 1950s to the 1960s. Solar radiation such as ultraviolet light can inactivate influenza viruses in the environment [21]. These seemingly non-infectious inactivated influenza viruses are in fact semiinfectious. Reassortment is the process by which influenza viruses, which carry RNA genomes comprising eight segments, exchange genetic material. For reassortment to occur, co-infection with two or more influenza virus strains is necessary. Semiinfectious particles deliver an incomplete set of viral genes to the cell, and therefore can support a full cycle of replication [22]. In this process, the influenza virus strain with pandemic potential and/or epidemic can be created.

\section{Possible extraterrestrial connection}

There is the additional, often ignored, effect of the circulating terrestrial virus hybridizing with compatible viruses or virions driven down to Earth from cometary sources due to sunspot effects. This model could fit the human outbreaks of EHF in Gabon and the Republic of Congo during 2001 to 2004 that consisted of multiple simultaneous epidemics caused by vastly different viral strains [23]. Another study revealed that the Ebola virus isolated from wild apes in the Gabon/Congo region were recombinants, and the recombination event probably occurred between 1996 and 2001 giving rise to a group of recombinant viruses that were responsible for a series of outbreaks in 2001-2003 [24]. In our view such recombinations could plausibly include hybridization with a RNA segment arriving from space.

Genetic data has suggested that the most recent Ebola outbreak in Sierra Leone was caused by a new strain evolving from two viral lineages from neighboring Guinea, and it was caused by a single interaction between humans and a viral reservoir in animals $[25,26]$. The latter animal reservoir may well have been replenished with RNA from space. The first case of EHF in Guinea occurred in December 2013 at the beginning of the dry season; this finding was consistent with observations from other countries that outbreaks often begin during the transition from the rainy season to drought [24,27-30]. Strong evidence points to fruit bats, which are present in large parts of West Africa, as the host species for the Ebola virus [31]. It is relevant that their swift and efficient mobility through the air enable them to sample large volumes of air, and if the air contains infalling virions, the bats could serve as crucible for the production of recombinant viruses that can trigger new pandemics.

The bats are highly mobile and seasonally nomadic in response to local food availability, and they probably shift habitats or territories because of changes in food availability or habitat suitability that may be influenced by drought weather. These bats may migrate from the depths of the rainforest and live closer to local villages in pursuit of food, sometimes amongst human populations. Fruit bats, a major 
source of protein, are widely eaten in rural West Africa. Presumably, local villagers might have had contact with bats or their secretions and then became infected with the Ebola virus through various human activities, such as hunting or handling of Ebola-infected bats.

Dry conditions can also cause fires and rainforest fragmentation. The latter increases the likelihood that bats will attempt to find other places to live. A prior outbreak investigation pointed to a coincident timing of an annual influx of migratory fruit bats in the Democratic Republic of Congo and the start of human Ebola outbreaks in local villages during 2007, and the first human case was linked to migratory bats that stayed in the area during the migratory season [32]. Furthermore, the new viral sequences also revealed that 2014 year's outbreak likely spread from Middle Africa through fruit bats within the last decade [33].

The lack of food resources caused by droughts can also increase interspecies contacts to compete for limited food sources available between the fruit bats and intermediate hosts, including chimpanzees and other primates, and these contacts may facilitate genetic recombination of any circulating Ebola virus in different species by sunspot activity. Finally, some Ebola viruses have successfully acquired the ability to spread from animal hosts to humans, triggering the outbreaks in humans.

\section{Forecasting future pandemics}

Although predicting the precise behavior of a sunspot cycle is not possible, approach to a maximum/minimum with an average 5.5 year period is relatively reliable [34]. So we can use sunspot data to provide early warning for epidemiological and virological surveillance of EHF as well as global influenza outbreaks. In addition, climatic factors, fruit bat migration, animal mortality, and serological and virological surveillance, should be conducted so that the risk of severe EHF outbreaks can be predicted or detected as early as possible.

We have seen that the onset of Ebola and Influenza pandemics appear to be remarkably coincident with extrema $(\mathrm{max} / \mathrm{min})$ of the sunspot cycle. Neither of these phases in the sunspot cycle is correlated strongly with the flux of cosmic rays or ionizing radiation at the ground level, so they are unlikely to play a direct role in genetic modifications at the Earth's surface. However, any viruses that may have been lofted in air currents into the stratosphere may be subject to mutagenic ionizing radiation, particularly at times of high solar activity. Additionally virions of cometary origin already present in the stratosphere that can interact with the genomes of terrestrially circulating viruses might be speedily brought down to Earth by changes in "space weather" that could be modulated through solar activity. These could include solar flares and coronal mass ejections.

Ramesh [13] has found evidence pointing to an offset between coronal mass ejections and sunspot maxima that can range from 3-5 years as shown in Figure 5. This would fit the conclusions we arrived at regarding the timing of pandemics, since peaks of major coronal mass ejections occur at sunspot minima \pm 1 year. It is worth noting also that individual large sunspots and their associated flare activity may well have a more significant effect on the Earth's electromagnetic environment than a large number of smaller sunspots. In November 2014, despite the low level of sunspot activity near maximum (weakest maximum in 100 years) a single individual sunspot the size of Jupiter was recorded. The sunspot numbers plotted in Figures 1 and 2 do not refer to individual large sunspots of this type, and such large spots near the minima in the sunspot cycle, along with a peak in the rate of coronal mass ejection, might play a role in the correlations we have found in the present paper.

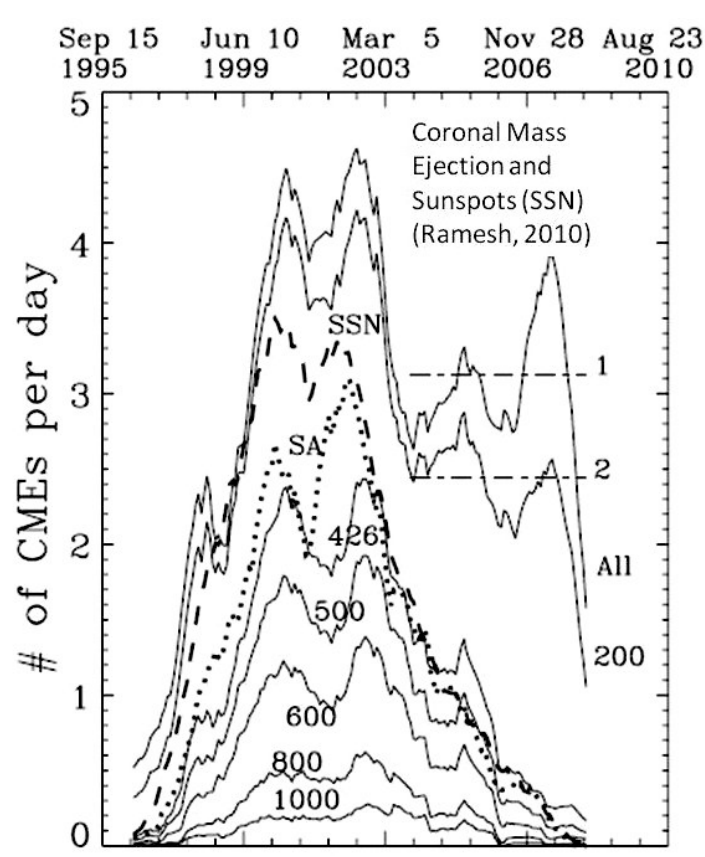

Figure 5: Coronal Mass Ejection events per day. Thin continuous lines represent the 13 point averages of $\mathrm{CR}$ averages of the CME rate (for widths greater than $30^{\circ}$ ) for lower cut off speeds 200, 452 (average speed), 500, 600, 800, and $1000 \mathrm{~km} . \mathrm{s}^{-1}$. The curve representing "All" corresponds to the CME rate of entire population.

If we accept that viruses/virions/microbes are continually introduced into the stratosphere by comets, their times of descent to the ground could be controlled by solar activity. Figure 6 shows the falling speed of spherical particles of various radii (density $1 \mathrm{~g} . \mathrm{cm}^{-3}$ ) through the standard atmosphere calculated by Kasten $[35,36]$. We see here that particles of viral size $a=0.01-0.03$ micrometres deposited by disintegrating cometary fragments at the top of the stratosphere $30 \mathrm{~km}$ will take 100 years to fall under gravity. Much faster descent would be enabled through electrical effects connected with solar activity. Large sunspots, solar flares and coronal mass ejection events would lead to large gradients of electrostatic potential in the Earth's atmosphere that could bring down viral sized particles in a matter of days rather than weeks.

\section{Appendix 1}

For analyzing data in Figure 3 the Chi-square test and nonconditional logistic regression were used to analyze the statistical association between sunspot extrema \pm one year and severe ebola outbreaks. The $\mathrm{P}$ values are two-sided, and all statistical analyses were conducted using SPSS software 15.0 (SPSS Inc., Chicago, IL, USA). The incidence of severe ebola outbreaks in sunspot extrema or \pm one year $(28.57 \%)$ was higher than those in other years $(5.56 \%)(\chi 2=3.40$, $\mathrm{P}=0.065)$. Logistic regression results showed that odds ratio value between sunspot extrema or \pm one year and severe Ebola outbreak is 6.80 (95\% CI, 0.73 to 63.11 ), and the difference had no statistical 
significance. If we use monthly mean sunspot numbers and the monthly onset of the disease events, the statistical results are the same as using yearly sunspot numbers (SSN). The difference had no statistical significance, probably due to the smallness of sample size.

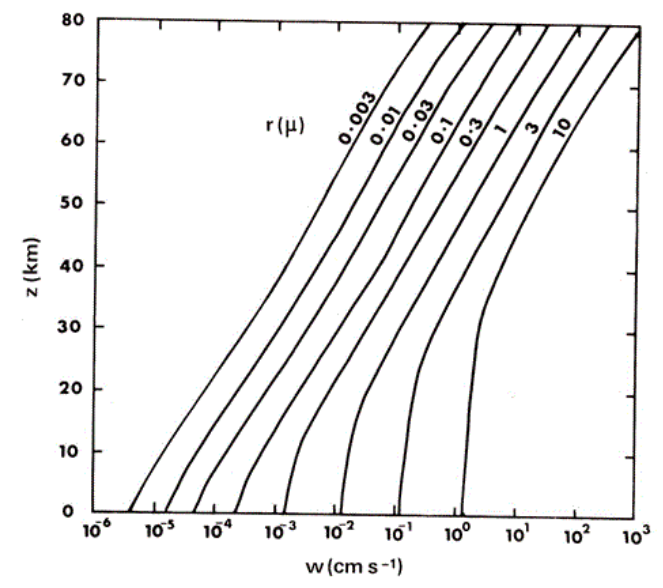

Figure 6: The falling speed of spherical particles of various radii and with density $1 \mathrm{~g} / \mathrm{cc}$.

We also notice that the 10 events are concentrated in solar cycles (SC) 23 and 24, but not in cycles 21 and 22. Multiple factors may contribute to EHF outbreaks in humans in the era of globalization. These diverse factors include environmental destruction, widespread deforestation, bushmeat consumption, climate change, economic underdevelopment, lack of medical resources, microbial genetic mutation, amongst others $[37,38]$. The vagaries of public health surveillance in developing countries must also be taken into account. It is entirely possible that public health departments may not have detected relevant outbreaks in cycles 21 and 22. This may be a task for further investigation.

\section{Conclusion}

In conclusion we stress that we cannot shirk our duty as scientists to continually monitor the various environmental parameters that might signal an impeding pandemic of influenza or other serious viral disease like EHF. High on our list of priorities is a continual microbiological surveillance of the stratosphere for the arrival of potentially threatening bacteria and viruses. In the event of a potential threat being discovered, preventative measures such as the production of appropriate vaccines could be put in place. In this way it might be possible to avert the worst consequences of any future pandemic that might threaten us.

\section{Acknowledgements}

We thank the following for providing data: Centers for Disease Control and Prevention (USA), Moscow Neutron Monitor (Russia), and Sunspot Index and Long-term Solar Observations, Royal Observatory of Belgium of World Center Data (Belgium).

\section{Author Contributions}

These authors contributed equally to this work.

\section{References}

1. Hoyle F, Wickramasinghe NC (1981) Evolution from Space, J M Dent Lond.

2. Wickramasinghe NC (2012) DNA sequencing and predictions of the cosmic theory of life. Astrophys Sp Sci 343: 1-5.

3. Bell EA, Boehnke P, Harrison T, Mao WL (2015) Potentially biogenic carbon preserved in a 4.1 billion-year-old zircon. PNAS 112: 14518-14521.

4. Altwegg $\mathrm{K}$, Balsiger $\mathrm{H}$, Bar-Nun A, Jean-Jacques B, Bieler AQ, et al. (2016) Prebiotic chemical - amino acid and phosphorus in the coma of comet 67P/Churyumov-Gerasimenko. Sci Adv 2: e1600285.

5. Harris MJ, Wickramasinghe NC, Lloyd D (2002) Detection of living cells in stratospheric samples. Proc. SPIE Int Soc Opt Eng 4495: 192.

6. Wainwright $\mathrm{M}$, Rose $\mathrm{CE}$, Baker AJ, Wickramasinghe NC, Omairi $\mathrm{T}$ (2015) Biological Entities Isolated from Two Stratosphere LaunchesContinued Evidence for a Space Origin. Astrobiol Outreach 3: 129.

7. Wainwright M, Omairi T (2016) New evidence that life is currently arriving to Earth from space. Edge Sci 25: 7-10.

8. Hoyle F, Wickramasinghe NC (1979) Diseases from Space, J.M. Dent and Son. London.

9. Qu J (2016) Is sunspot activity a factor in influenza pandemics? Rev Med Virol.

10. Hoyle F, Wickramasinghe NC (1961) A Note on the Origin of the Sun's Polar Field. Mon Not RAS 123: 51-59.

11. Weinstein L (1976) Influenza - 1918, a Revisit? New Engl J Med 294: 1058-1060.

12. Hope-Simpson RE (1978) Sunspots and flu: a correlation. Nature 275: 86.

13. Ramesh KB (2010) Coronal mass ejections and sunspots - solar cycle perspective. Astrophys J Lett 712: L77-L80.

14. O'Sullivan D (2007) Exposure to galactic cosmic radiation and solar energetic particles. Radiat Prot Dosim 125: 407-411.

15. Hathaway DH, Wilson RM (2004)What the sunspot record tells us about space climate. Solar Phys 224: 5-19.

16. Domingo E (2010) Mechanisms of viral emergence. Vet Res 41: 38.

17. Subbarao K, Klimov A, Katz J, Regnery H, Lim W, et al. (1998) Characterization of an avian influenza A ( $\mathrm{H} 5 \mathrm{~N} 1)$ virus isolated from a child with a fatal respiratory illness. Science 279: 393-396.

18. Guan Y, Shortridge KF, Krauss S, Webster RG (1999) Molecular characterization of H9N2 influenza viruses: were they the donors of the "internal" genes of H5N1 Viruses in Hong Kong? Proc Natl Acad Sci USA 96: 9363-9367.

19. Liu D, Shi W, Shi Y, Wang D, Xiao H, et al. (2013) Origin and diversity of novel avian influenza A H7N9 viruses causing human infection: phylogenetic, structural, and coalescent analyses. Lancet 381: 1926-1932.

20. Lam TT, Wang J, Shen Y, Zhou B, Duan L, et al. (2013) The genesis and source of the H7N9 influenza viruses causing human infectionsin China. Nature 502: 241-244.

21. Sagripanti JL, Lytle CD (2007) Inactivation of influenza virus by solar radiation. Photochem Photobiol 83: 1278-1282.

22. Fonville JM, Marshall N, Tao H, Steel J, Lowen AC (2015) Influenza Virus Reassortment is enhanced by semi-infectious particles but can be suppressed by defective interfering particles. PLoS Pathog 11: e1005204.

23. Leroy EM, Rouquet P, Formenty P, Souquière S, Kilbourne A, et al. (2004) Multiple Ebola virus transmission events and rapid decline of central African wildlife. Science 303: 387-390.

24. Wittmann TJ, Biek R, Hassanin A, Rouquet P, Reed P, et al. (2007) Isolates of Zaire ebolavirus from wild apes reveal genetic lineage and recombinants. Proc Natl Acad Sci USA 104: 17123-17127.

25. Baize S, Pannetier D, Oestereich L, Rieger T, Koivogui L, et al. (2014) Emergence of Zaire Ebola virus disease in Guinea. N Engl J Med 371: 1418-1425. 
Citation: Qu J, Gao Z, Zhang Y, Wainwright M, Wickramasinghe NC, et al. (2016) Sunspot Activity, Influenza and Ebola Outbreak Connection. Astrobiol Outreach 4: 154. doi:10.4172/2332-2519.1000154

Page 7 of 7

26. Gire SK, Goba A, Andersen KG, Sealfon RSG, Park DJ, et al. (2014) Genomic surveillance elucidates Ebola virus origin and transmission during the 2014 outbreak. Science 345: 1369-1372.

27. Lahm SA, Kombila M, Swanepoel R, Barnes RF (2007) Morbidity and mortality of wild animals in relation to outbreaks of Ebola haemorrhagic fever in Gabon, 1994-2003. Trans R Soc Trop Med Hyg 101: 64-78.

28. Bermejo M, Rodríguez-Teijeiro JD, Illera G, Barroso A, Vilà C, et al. (2006) Ebola outbreak killed 5000 gorillas. Science 314: 1564.

29. Pinzon JE, Wilson JM, Tucker CJ, Arthur R, Jahrling PB, et al. (2004) Trigger events: enviroclimatic coupling of Ebola hemorrhagic fever outbreaks. Am J Trop Med Hyg 71: 664-674.

30. Jezek Z, Szczeniowski MY, Muyembe-Tamfum JJ, McCormick JB, Heymann DL (1999) Ebola between outbreaks: intensified Ebola hemorrhagic fever surveillance in the Democratic Republic of the Congo, 1981-1985. J Infect Dis 179: S60-S64.

31. Leroy EM, Kumulungui B, Pourrut X, Rouquet P, Hassanin A, et al. (2005) Fruit bats as reservoirs of Ebola virus. Nature 438: 575-576.

32. Leroy EM, Epelboin A, Mondonge V, Pourrut X, Gonzalez JP, et al. (2009) Human Ebola outbreak resulting from direct exposure to fruit bats in
Luebo, Democratic Republic of Congo, 2007. Vector Borne Zoonotic Dis 9: 723-728.

33. Vogel G (2014) Genomes reveal start of Ebola outbreak. Science 345: 989-990.

34. Ng KK (2016) Prediction Methods in Solar Sunspots Cycles. Sci Rep 6: 21028.

35. Kasten F (1968) Falling speed of aerosol particles. Journal of Applied Meteorology 7: 944-947.

36. Zhang G, Shohan D, Gilichinsky D, Davydov S, Castello JD, et al. (2006) Evidence of influenza A virus RNA in Siberian lake ice. J Virol 80: 12229-122351.

37. Bausch DG, Schwarz L (2014) Outbreak of ebola virus disease in Guinea: where ecology meets economy. PLoS Negl Trop Dis 8: e3056.

38. Ng S, Basta NE, Cowling BJ (2014) Association between temperature, humidity and ebolavirus disease outbreaks in Africa, 1976 to 2014. Euro Surveill 19: 1-11. 